\title{
GASEIFICAÇÃO DA CASCA DE ARROZ USANDO CATALISADORES (Ni, Co)/HBETA E (Ni, Co)/HZSM-5
}

\author{
A. P. N. MOURA, J. A. C. SILVA, I. C. S. COSTA e M. S. BATISTA \\ Universidade Federal de São João Del Rei, Departamento de Engenharia Química \\ E-mail: marcelobatista@ufsj.edu.br
}

\begin{abstract}
RESUMO - A gaseificação da casca de arroz é uma alternativa sustentável para obtenção de gás de síntese e produção de biocombustíveis. Esse trabalho tem como objetivo avaliar o desempenho dos catalisadores de níquel e cobalto suportados nas zeólitas HZSM-5 e HBETA na reação de gaseificação da casca de arroz com vapor de água. Os catalisadores foram preparados pelo método de impregnação e foram caracterizados por difratometria de raios $X(D R X)$ e a redução com hidrogênio à temperatura programada $\left(\mathrm{RTP}-\mathrm{H}_{2}\right)$. Os resultados mostraram que a temperatura e o tipo de zeólita influenciam na composição da mistura gasosa na gaseificação da casca de arroz pirolisada. Em baixas temperaturas $\left(500^{\circ} \mathrm{C}\right)$ se observa maiores porcentagens de $\mathrm{CH}_{4}$ e uma diminuição desse gás com o aumento da temperatura, bem como o favorecimento da formação de $\mathrm{H}_{2}$. A atividade na gaseificação da casca de arroz baseada na produção de $\mathrm{H}_{2}$ foi: 10Ni/HZSM-5>10Co/HZSM-5 > 10Ni/HBETA > 10Co/HBETA.
\end{abstract}

\section{INTRODUÇÃO}

Os combustíveis fósseis vêm sendo amplamente utilizados para suprir a demanda energética mundial, com isso, observou-se uma grande quantidade de poluentes atmosféricos sendo emitidos diariamente, trazendo sérios prejuízos ambientais e também à saúde da população (Shahbaz et al., 2017). Na tentativa de diminuir a emissão de gases poluentes, novas formas de obtenção de energias renováveis tem sido desenvolvidas. A gaseificação de biomassa para a produção de gás de síntese aparece como uma alternativa de fonte de energia renovável que pode contribuir com a matriz energética mundial, além de diminuir a quantidade de resíduos sólidos urbanos e agrícolas (Vandamme et al., 2013).

A viabilidade econômica da gaseificação de biomassa tem se destacado entre outras possíveis rotas de obtenção de energia renovável. O gás de síntese obtido na gaseificação de biomassa pode ser convertido em combustível líquido, ou seja, pode ser utilizado como matéria prima para a produção de: etanol, metanol e outros hidrocarbonetos (Huber et al., 2006). A composição do gás de síntese pode variar de acordo com o tipo de biomassa e o agente de gaseificação empregado, mas consiste principalmente de hidrogênio $\left(\mathrm{H}_{2}\right)$, monóxido de carbono (CO), dióxido de carbono $\left(\mathrm{CO}_{2}\right)$ e metano $\left(\mathrm{CH}_{4}\right)$. De acordo com estudos realizados, o uso da água como agente de gaseificação apresenta vantagens sobre outros agentes, como o oxigênio, devido à menor produção de alcatrão e ao maior rendimento em gás (Dai et al., 2015; Hosseini et al., 2012). 
As reações envolvidas no processo de gaseificação podem ser diretamente influenciadas pelo uso de catalisadores, pois estes podem modificar a concentração dos produtos gasosos e aumentar a velocidade das reações em temperaturas mais baixas. Por esse motivo, um dos desafios desta rota é encontrar um catalisador que possua baixo custo de preparação e que seja ativo nas reações desejadas. Os catalisadores a base de níquel apresentam alta eficiência na gaseificação, principalmente quando o sistema é operado em altas temperaturas, pois diminui a formação de metano. Os catalisadores a base cobalto também se mostraram ativos na gaseificação a vapor da madeira e outras biomassas (Sornkade et al., 2013; Li et al., 2011). O uso das zeólitas HZSM-5 e HBETA podem aumentar a dispersão de óxidos de níquel e de cobalto, e consequentemente, aumentar a produção do gás de síntese (Kimura et al., 2006).

Neste contexto, esse trabalho tem como objetivo produzir o gás de síntese através da gaseificação da casca de arroz utilizando catalisadores a base de níquel e de cobalto suportados nas zeólitas HZSM-5 e HBETA para estudar a influência da temperatura e do suporte zeolítico na composição do gás.

\section{METODOLOGIA}

\section{Preparação dos Catalisadores}

As zeólitas HZSM-5 e HBETA foram preparadas através de três trocas iônicas consecutivas de 8 h, utilizando zeólitas ZSM-5 e BETA comerciais e uma solução de ácido clorídrico $0,1 \mathrm{~mol} / \mathrm{L}$. Utilizou-se a proporção de $50 \mathrm{~mL}$ de solução ácida por cada grama de zeólita. Após as trocas iônicas, calcinou-se os materiais a $600^{\circ} \mathrm{C}$ por $2 \mathrm{~h}\left(10^{\circ} \mathrm{C} / \mathrm{min}\right)$. Os catalisadores 10Co/HBETA, 10Co/HZSM-5, 10Ni/HBETA e 10Ni/HZSM-5 contendo 10\% $(\mathrm{m} / \mathrm{m})$ de metal foram preparados por impregnação incipiente utilizando-se nitrato hexahidratado de cobalto $\left(\mathrm{Co}\left(\mathrm{NO}_{3}\right)_{2} \cdot 6 \mathrm{H}_{2} \mathrm{O}\right)$ e o cloreto de níquel $\left(\mathrm{NiCl}_{2} \cdot 6 \mathrm{H}_{2} \mathrm{O}\right)$. Em seguida, os catalisadores foram calcinados a $600^{\circ} \mathrm{C}$ por $2 \mathrm{~h}\left(10^{\circ} \mathrm{C} / \mathrm{min}\right)$.

\section{Caracterização dos Catalisadores}

Os catalisadores foram caracterizados por difratometria de raios $\mathrm{X}$ (DRX) e redução com hidrogênio a temperatura programada $\left(\mathrm{RTP}-\mathrm{H}_{2}\right)$. Nas análises de DRX foi utilizado o método do pó e um difratômetro Shimadzu (modelo XRD 6000) que operou com radiação monocromática $\mathrm{Cu}-\mathrm{K} \alpha$, com tensão de $30 \mathrm{KV}$ e corrente de $30 \mathrm{~mA}$. A velocidade do goniômetro foi de $2^{\circ}(2 \theta) / \mathrm{min}$, com variação do ângulo na faixa de $10^{\circ}$ a $80^{\circ}(2 \theta)$. As análises de RTP- $\mathrm{H}_{2}$ foram realizadas em um equipamento SAMP3 (Termolab equipamentos Ltda.) com um detector de condutividade térmica. Em cada análise utilizou-se um fluxo de 30 $\mathrm{mL} / \mathrm{min}$ de gás $\left(2 \% \mathrm{H}_{2} / \mathrm{Ar}\right)$. A amostra foi submetida ao fluxo gasoso e foi aquecida da temperatura ambiente até $1000^{\circ} \mathrm{C}\left(10^{\circ} \mathrm{C} / \mathrm{min}\right)$.

\section{Preparação da Casca de Arroz}

Utilizando-se um reator de aço inox com capacidade de $280 \mathrm{~mL}$, pirolisou-se $30 \mathrm{~g}$ de casca de arroz sob pressão atmosférica. A remoção do oxigênio do reator foi realizada através de uma vazão de arraste de $50 \mathrm{~mL} / \mathrm{min}$ de gás nitrogênio $(99,999 \%)$. O reator foi aquecido até $400^{\circ} \mathrm{C}\left(10^{\circ} \mathrm{C} / \mathrm{min}\right)$ e foi mantido nesta temperatura por $5 \mathrm{~min}$. $\mathrm{Na}$ medição da temperatura 
utilizou-se um termopar e o reator foi envolvido por lã de rocha para melhorar o isolamento térmico.

\section{Avaliação Catalítica}

A gaseificação foi realizada em uma unidade multipropósito de reação, na qual $10 \mathrm{mg}$ de catalisador e $100 \mathrm{mg}$ de casca de arroz pirolisada foram adicionados ao reator de aço inox ( $d=1 / 4$ " e $\mathrm{L}=30 \mathrm{~cm}$ ) com um leito feito de lã de quartzo. O reator foi inserido em um forno com controlador PID de temperatura. Nos ensaios foi utilizado um fluxo de $40 \mathrm{~mL} / \mathrm{min}$ de gás hélio que passou por um saturador contendo água aquecida a $45^{\circ} \mathrm{C}$ por um banho termostático. O reator foi aquecido da temperatura ambiente até $900^{\circ} \mathrm{C}\left(10^{\circ} \mathrm{C} / \mathrm{min}\right)$ e os gases produzidos na reação foram analisados por um espectrômetro de massas (THERMO) acoplado à linha de testes. Os gases analisadas foram $\mathrm{H}_{2}, \mathrm{CO}, \mathrm{CO}_{2}, \mathrm{CH}_{4}, \mathrm{H}_{2} \mathrm{O}$ e $\mathrm{He}$.

\section{RESULTADOS E DISCUSSÃO}

$\mathrm{Na}$ Figura 1 são apresentados os difratogramas de raios $\mathrm{X}$ dos catalisadores 10Co/HBETA, 10Co/HZSM-5, 10Ni/HBETA e 10Ni/HZSM-5. Note que os difratogramas apresentaram picos característicos da zeólita ZSM-5 em $2 \theta=23,1^{\circ}$ e $24,4^{\circ}$ e da zeólita BETA em $2 \theta=22,3^{\circ}$.

Figura 1 - DRX dos catalisadores (a) 10Co/HBETA e 10Co/HZSM-5; (b)10Ni/HBETA e 10Ni/HZSM-5. Picos ( $\square) \mathrm{NiO},(\boldsymbol{\bullet}) \mathrm{Co}_{3} \mathrm{O}_{4},(*)$ BETA e ( $\left.\boldsymbol{\Delta}\right)$ ZSM-5.
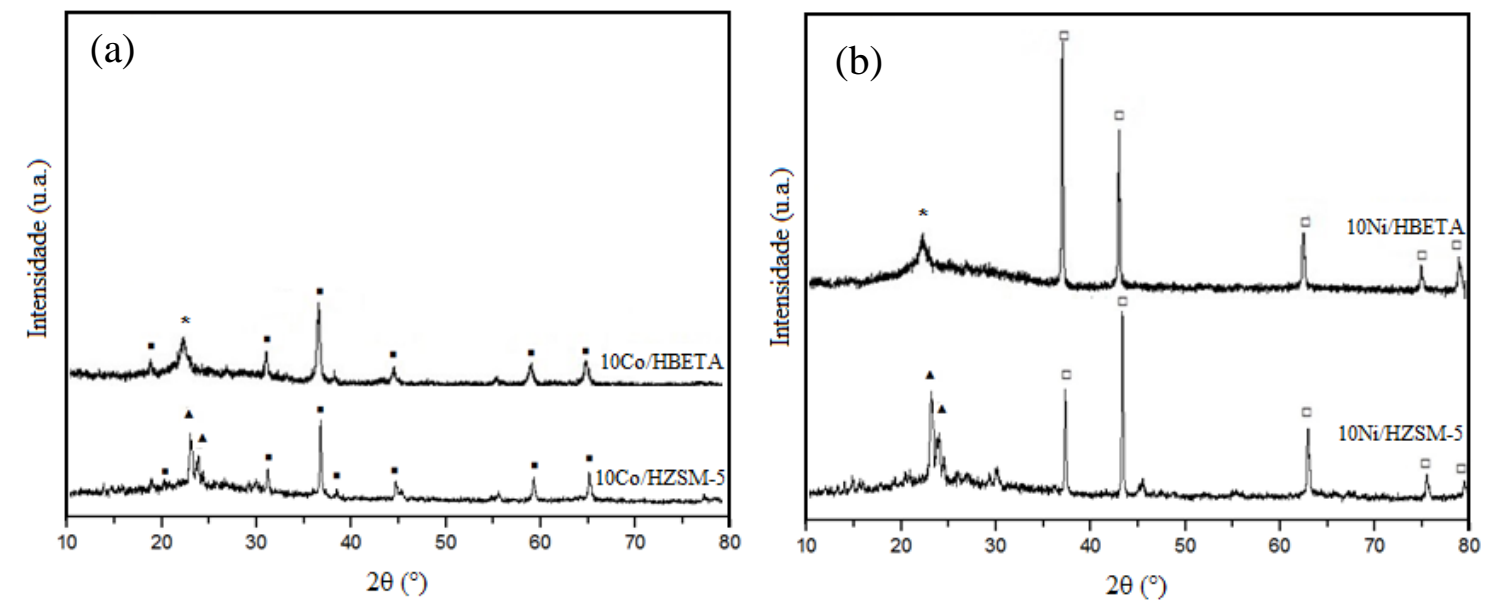

Nos difratogramas dos catalisadores 10Ni/HBETA e 10Ni/HZSM-5 nota-se picos referentes ao óxido de níquel $(\mathrm{NiO})$, observados em $2 \theta=37,3^{\circ}, 43,3^{\circ}, 62,9^{\circ}, 65,4^{\circ}, 79,4^{\circ}$ (Ficha $\mathrm{n}^{\circ}$ 78-0423 JCPDS, 1997). Nos difratogramas dos catalisadores 10Co/HBETA e 10Co/HZSM-5 os principais picos identificados foram atribuídos ao óxido de cobalto $\left(\mathrm{Co}_{3} \mathrm{O}_{4}\right)$ em $2 \theta=18,9^{\circ}, 31,3^{\circ}, 36,8^{\circ}, 38,5^{\circ}, 44,8^{\circ}, 59,3^{\circ}$ e $65,2^{\circ}$ (Ficha $n^{\circ} 80-1541$ JCPDS, 1994).

Na Figura 2 são apresentados os perfis de RTP-H $\mathrm{H}_{2}$ dos catalisadores 10Co/HBETA, 10Co/HZSM-5, 10Ni/HBETA e 10Ni/HZSM-5. Os catalisadores 10Co/HZSM-5 e 10Co/HBETA apresentaram dois picos que podem ser associados à redução do óxido de cobalto em duas etapas, sendo a primeira etapa de $\mathrm{Co}_{3} \mathrm{O}_{4}$ a $\mathrm{CoO}$ e a segunda etapa de $\mathrm{CoO}$ a $\mathrm{Co}^{0}$ (TIERNAN et al., 2001). O ombro observado na faixa de 371 a $450^{\circ} \mathrm{C}$ para o catalisador 
10Co/HBETA corresponde à interação do óxido de cobalto com o suporte (ZANG et al., 2011).

Figura 2 - Perfil de RTP-H ${ }_{2}$ dos catalisadores (a) 10Co/HBETA e 10Co/HZSM-5;

\section{(b)10Ni/HBETA e 10Ni/HZSM-5.}
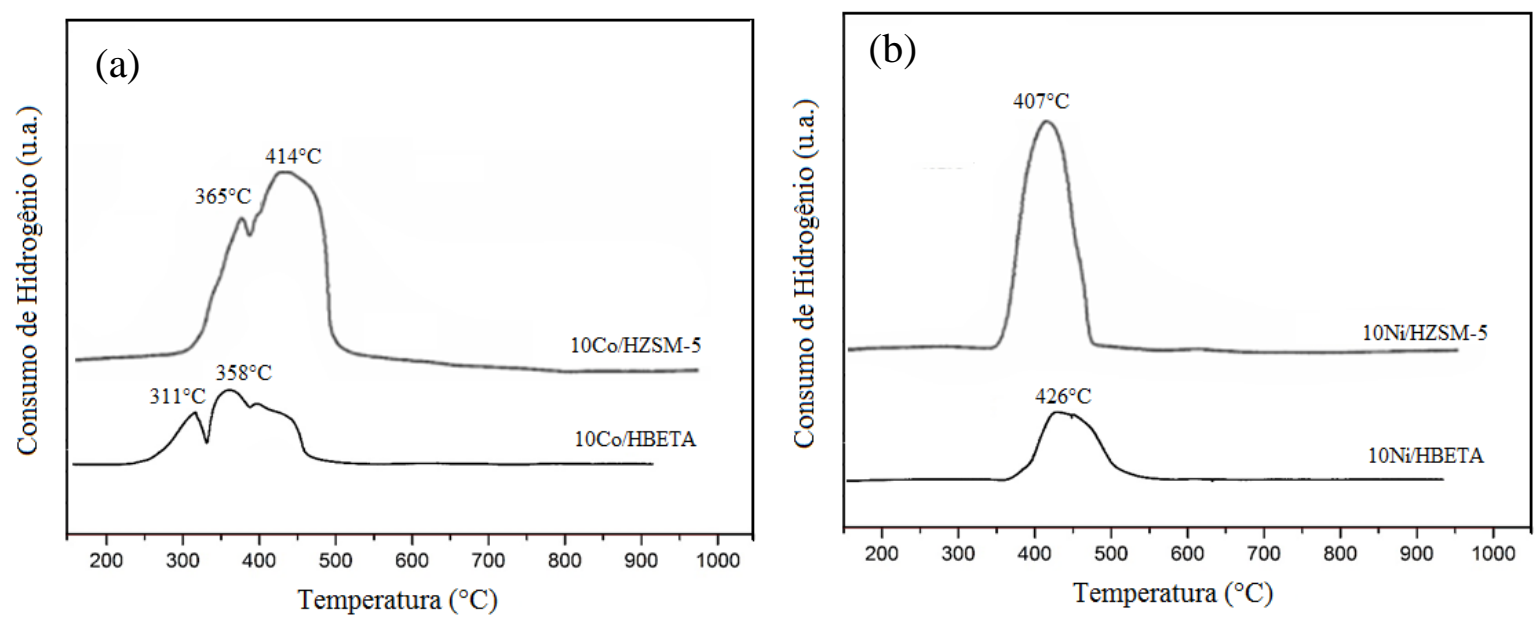

O perfil dos catalisadores 10Ni/HBETA e 10Ni/HZSM-5 apresentam apenas um pico de redução, mostrando que a redução ocorre em uma única etapa. Esse pico corresponde a redução de $\mathrm{NiO}$ a $\mathrm{Ni}^{0}$, em $407^{\circ} \mathrm{C}$ para $10 \mathrm{Ni} / \mathrm{HZSM}-5$ e em $426^{\circ} \mathrm{C}$ para $10 \mathrm{Ni} / \mathrm{HBETA}$. Esse resultado esta de acordo com a faixa de temperatura esperada para a redução do óxido de níquel, entre $400^{\circ} \mathrm{C}$ e $450^{\circ} \mathrm{C}$ (ZHANG et al., 2012).

Na Figura 3 são apresentados os principais produtos da gaseificação nas temperaturas de $500^{\circ} \mathrm{C}, 600^{\circ} \mathrm{C}$ e $700^{\circ} \mathrm{C}$, para os catalisadores 10Co/HBETA, 10Co/HZSM-5, 10Ni/HBETA e 10Ni/HZSM-5. Note que a concentração de $\mathrm{H}_{2}, \mathrm{CO}, \mathrm{CO}_{2}$ e $\mathrm{CH}_{4}$ apresentam variações conforme o aumento da temperatura devido às reações térmicas envolvidas na gaseificação e algumas delas são concorrentes.

Figura 3 - Composição da mistura gasosa na gaseificação catalítica da casca de arroz.

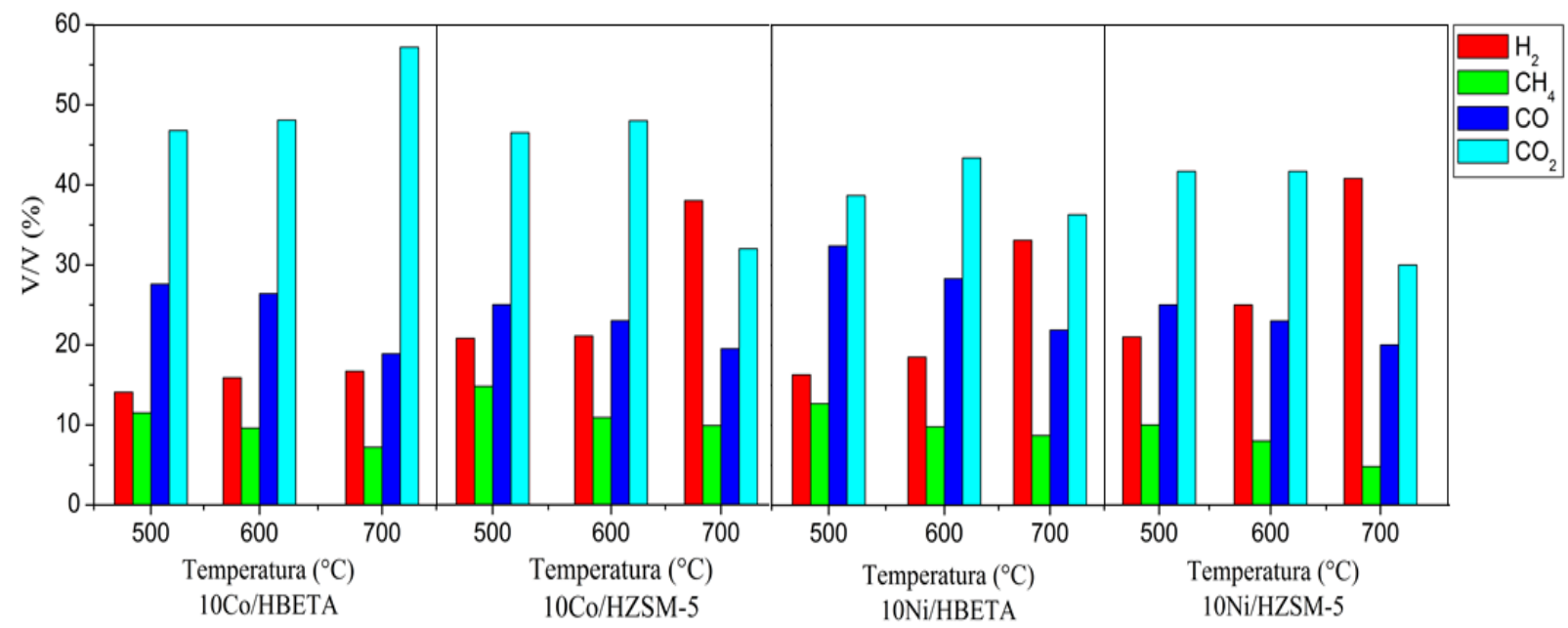


Na Figura 3 pode-se observar que a concentração de metano é maior nas temperaturas mais baixas. Isso pode estar associado à ocorrência da reação metanação, conforme Equação 1. No entanto, note que o aumento da temperatura faz com que a porcentagem de $\mathrm{CH}_{4}$ diminua, pois, o caminho inverso dessa reação, conhecida como reforma do metano, passa a acontecer em uma velocidade maior, contribuindo com o aumento da porcentagem de $\mathrm{CO}$ e $\mathrm{H}_{2}$.

$$
3 \mathrm{H}_{2}+\mathrm{CO} \rightarrow \mathrm{CH}_{4}+\mathrm{H}_{2} \mathrm{O}
$$

O aumento da produção de hidrogênio nas temperaturas superiores a $700^{\circ} \mathrm{C}$ está associado à reação de vapor de água fornecido via saturador e o carbono da casca de arroz, conforme Equação 2. A termodinâmica desta reação é favorecida em temperaturas acima de $630^{\circ} \mathrm{C}$ (SMITH et al., 2007).

$$
\mathrm{C}+\mathrm{H}_{2} \mathrm{O} \rightarrow \mathrm{H}_{2}+\mathrm{CO}
$$

$\mathrm{Na}$ Figura 3, entre os catalisadores de cobalto, 10Co/HZSM-5 produziu a maior porcentagem de $\mathrm{H}_{2}$, quando comparado a 10Co/HBETA. No entanto, observa-se que este último foi mais propenso à formação de $\mathrm{CO}_{2}$. Esse resultado pode ser associado à ocorrência da reação WGS (Water Gas Shift) que é favorecida na presença de vapor de água, conforme Equação 3.

$$
\mathrm{H}_{2} \mathrm{O}+\mathrm{CO} \rightarrow \mathrm{CO}_{2}+\mathrm{H}_{2}
$$

A Figura 3 mostra que 10Ni/HZSM-5 produziu o maior porcentagem de $\mathrm{H}_{2}$ e a menor de $\mathrm{CH}_{4}$. Note o catalisador Co/HZSM-5 também alcançou resultado superior aos catalisadores de níquel e cobalto suportados em HBETA, indicando que o uso da zeólita ZSM-5 como suporte favoreceu a produção de $\mathrm{H}_{2}$. Assim, os catalisadores apresentaram seguinte ordem de seletividade a $\mathrm{H}_{2}$ : 10Ni/HZSM-5> Co/HZSM-5> 10Ni/HBETA>10Co/HBETA.

\section{CONCLUSÕES}

De acordo com os resultados apresentados pode-se concluir que os catalisadores aumentaram a velocidade das reações envolvidas na gaseificação, modificando a concentração de $\mathrm{H}_{2}, \mathrm{CO}, \mathrm{CO}_{2}$ e $\mathrm{CH}_{4}$. Foi observado que a temperatura também influencia fortemente a composição da mistura gasosa na gaseificação da casca de arroz pirolisada. Em baixas temperaturas $\left(500^{\circ} \mathrm{C}\right)$ se observa maiores porcentagens de $\mathrm{CH}_{4}$ e uma diminuição desse gás com o aumento da temperatura, bem como o favorecimento da formação de $\mathrm{H}_{2}$. A atividade na gaseificação da casca de arroz pirolisada baseada na produção de $\mathrm{H}_{2}$ foi: $10 \mathrm{Ni} / \mathrm{HZSM}-$ 5>10Co/HZSM-5>10Ni/HBETA>10Co/HBETA. A zeólita ZSM-5 apresentou o melhor desempenho como suporte para cobalto e níquel no processo de gaseificação.

\section{REFERÊNCIAS}

DAI, J.; SAAYMAN, J.; GRACE, J. R.; ELLIS, N. Gasification of woody biomass. Annu Rev. Chem, Biomol. Eng. V. 6, p. 77-99, 2015. 
HOSSEINI, M.; DINCER, I.; ROSEN,M. A. Steam and air fed biomass gasification: comparisons based on energy and exergy Int. J. Hydrog. Energy. V. 37, p. 16446 16452. 2012.

HUBER, G. W.; IBORRA, S.; CORMA, A. Synthesis of transportation fuels from biomass: chemistry catalysts, and engineering. Chem. Rev. v. 106, p. 4044 - 4098, 2006.

KIMURA, T.; MIYAZAWA, T.; NISHIKAWA, T.; KADO, J.; OKUMURA, K.; MIYAO, T. Development of Ni catalysts for tar removal by steam gasification of biomass. Applied Catalysis. V. 68, p. $160-170,2006$.

LI, S.; LU, Y.; GUO, L.; ZHANG, X. Hydrogen production by biomass gasification in supercritical water with bimetallic $\mathrm{Ni}-\mathrm{M} / \gamma \mathrm{Al}_{2} \mathrm{O}_{3}$ catalystis $(\mathrm{M}=\mathrm{Cu}, \mathrm{Co}$ and $\mathrm{Sn})$ Int. $J$. Hydrogen Energy. V. 36, p. 14391-14400, 2011.

SHAHBAZ, M.; YUSUP, S.; INAYAT, A.; PATRICK, O.; AMMA, M. The influence of catalysts in biomassa steam gasification and catalytic potential of coal bottom ash in biomass steam gasification: A review. Renewable and Sustainable Energy Reviews. V. 73, p. 468-476, 2017.

SMITH, J. M.; VAN NESS,H. C.; ABBOTT, M. M. Introdução à Termodinâmica da Engenharia Química, LTC, Rio de Janeiro, 2007.

SORNKADE, P.; ATONG, D.; SRICHAROENCHAIKUL, V. Enhancement of Cassava Rhizome Gasification Using Mono-Metallic Cobalt catalysts. Energy Procedia. 2013, 34, 273-281.

TIERNAN, M. J.; BARNES, P. A.; PARKERS, G. M. B. Reduction of iron oxide catalysts: the investigation of kinetic parameters using rate perturbation and linear heating thermoanalytical techniques. J. Phys. Chem. B. 2001, 205, 220-228.

VANDAMME, D.; FOUBERT, I; MUYLAERT, K. Flocculation as a low-cost method for harvesting microalgae for bulk biomass production. Trends Biotechnology. 2013, 31, 233-239.

ZHANG, L.; DONG, L.; YU, W.; LIU, L.; DENG, Y.; LIU, B.; WAN, H.; GAO, F.; SUN, K.; DONG, L. Effect of cobalt precursors on the dispersion, reduction, and CO oxidation of $\mathrm{CoOx} / \gamma-\mathrm{Al}_{2} \mathrm{O}_{3}$ catalysts calcined in $\mathrm{N}_{2}$. Journal of Colloid and Interface Science. 2011, 355, 464-471.

ZHANG, Q.; WANG, T.; LI, B.; JIANG, T.; MA, L.; ZHANG, X.; LIU, Q. Highly selective sorbitol hydrogenolysis to liquid alkanes over Ni/HZSM-5 catalysts modified with pure silica MCM-41. Applied Energy, 2012, 97, 509-513.

ZHAO, Z.; LAKSHMINARAYANAN, N.; SWARTZ, S. L.; ARKENBERG. G. B.; FELIX, L. G.; SLIMANE, R. B.; CHOI, C. C.; OZKAN, U. S. Characterization of olivinesupported nickel silicate as potential catalysts for tar removal from biomass gasification, Appl Catal A: Gen. 2015, 489, 42-50 